\title{
Classification of perturbations of the hydrogen atom by small static electric and magnetic fields
}

\author{
B y K. Efstathiou ${ }^{1}$, D. A. SAdovskiÍ ${ }^{2} \dagger$, And B. I. ZhilinskiÍ ${ }^{2}$ \\ ${ }^{1}$ Instituut voor Wiskunde en Informatica, Rijksuniversiteit Groningen, \\ Groningen 9747 AC, The Netherlands \\ ${ }^{2}$ UMR du CNRS 8101, Université du Littoral, 59140 Dunkerque, France
}

We consider perturbations of the hydrogen atom by sufficiently small homogeneous static electric and magnetic fields of all possible mutual orientations. Normalising with regard to the Keplerian symmetry, we uncover resonances and conjecture that the parameter space of this family of dynamical systems is stratified into zones centred on the resonances. The 1:1 resonance corresponds to the orthogonal field limit, studied earlier by Cushman \& Sadovskií (2000). We describe the structure of the 1:1 zone, where the system may have monodromy of different kinds, and consider briefly the 1:2 zone.

Keywords: perturbed Kepler system, singular reduction, energy-momentum map, monodromy

\section{Introduction}

Perturbations of the hydrogen atom by external electric and magnetic fields constitute one of the most fundamental families of atomic physics systems. In the limit of the infinite proton mass and with spin and relativistic corrections neglected, this family becomes a quantum realisation of a specific class of perturbations of the Kepler system with Hamiltonian (in atomic units)

$$
H=\frac{1}{2} \mathbf{P}^{2}-\frac{1}{|\mathbf{Q}|}+F_{e} Q_{2}+F_{b} Q_{1}+\frac{G}{2}\left(Q_{2} P_{3}-Q_{3} P_{2}\right)+\frac{G^{2}}{8}\left(Q_{2}^{2}+Q_{3}^{2}\right)=E,
$$

where $(\mathbf{Q}, \mathbf{P})$ are standard canonical coordinates on the phase space $\mathbb{R}^{6}$ and 3-vectors $\mathbf{F}=\left(F_{b}, F_{e}, 0\right)$ and $\mathbf{G}=(G, 0,0)$ represent the electric and the magnetic field, respectively. We remain at sufficiently large negative physical energy $E$ and consider bounded motion near the origin. For sufficiently small fields, we can use the well-known dynamical Keplerian symmetry SO(4) of the unperturbed system and consider the angular momentum $\mathbf{L}$ and the eccentricity vector $\mathbf{K}$ as approximate integrals of motion. The Hamiltonian (1.1) can be first regularised and then normalised with respect to the action of this symmetry, which is defined by the flow of the regularised unperturbed system. Using such transformation, we can replace the original non-integrable system with three degrees of freedom described by (1.1) by an integrable approximation. More specifically, we obtain a three-parameter family of integrable dynamical models with parameters $\left(F_{b}, F_{e}, G\right)$. By analysing and characterising the qualitatively different member systems in this family, we can classify the real non-integrable dynamical systems with Hamiltonian (1.1).

$\dagger$ Author for correspondence (sadovski@univ-littoral.fr) 

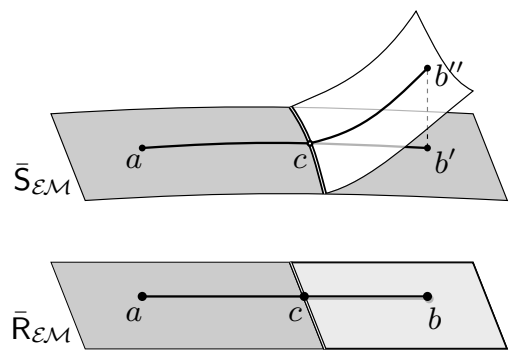

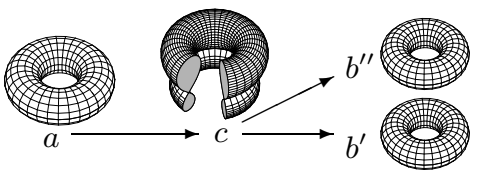

Figure 1. Example of overlapping lower cells in the 2D-image of an energy-momentum map $\mathcal{E M}$ (bottom left) and its two-sheet cell unfolding surface (top left). Points $a, b^{\prime}, b^{\prime \prime}$, and $c$ lift each to a connected component (right); $b^{\prime}$ and $b^{\prime \prime}$ correspond to the same $\mathcal{E M}$ value $b$. Double line marks branching boundary; bold solid line marks a path connecting $a, c$ and $b$.

The result of the reduction is a two degree of freedom Hamiltonian system described by the Hamiltonian

$$
H_{n}=2 n+n\left(H_{1}+\cdots\right)
$$

where $n$ is the value of the Keplerian integral of motion $N$; in the quantum system, $n$ corresponds to the principal quantum number, and $H_{n}$ describes the internal structure of $n$-shells. Furthermore, the flow of $H_{1}$ is linear and is characterized by two frequencies $\omega_{+}$ and $\omega_{-}$that depend on the external parameters $\left(F_{b}, F_{e}, G\right)$ of the system.

It follows that we can obtain an integrable approximation by normalising a second time. This specifics of systems with Hamiltonian (1.1) was exploited by Pauli (1926), cf. (van der Waerden, 1968; Valent, 2003). Note that instead of normalising with respect to the flow of the vector field of the Hamiltonian function $H_{1}$, we can choose an $\mathbb{S}^{1}$ flow given by the vector field of a momentum $\mu$ which Poisson commutes with $H_{1}$, and which is chosen typically so that $\omega \mu \approx H_{1}$ with $\omega=\omega\left(G, F_{e}, F_{b}\right)>0$ a constant. The rational frequencies $\omega k_{ \pm}$of $\mu$ where $k_{ \pm} \in \mathbb{Z}_{>0}$ approximate the frequencies $\omega_{ \pm}$of $H_{1}$; the small difference $H_{1}-\omega \mu$ is called linear detuning term. See $\S 3$ and $\S 4$ for concrete choices of $\omega$ and $\mu$ respectively. Thus any perturbation of the hydrogen atom by sufficiently small static external fields possesses a resonant integrable approximation with first integrals $N$ (Keplerian action), $\mu$ (momentum), and $\mathcal{H}$ (second reduced energy) with respective values $n \geq 0, m$, and $h$. We can now attempt to characterise the entire family of perturbations of the hydrogen atom by sufficiently small static external fields on the basis of the qualitative description of the family of such approximations for each resonance $k_{+}: k_{-}$. 

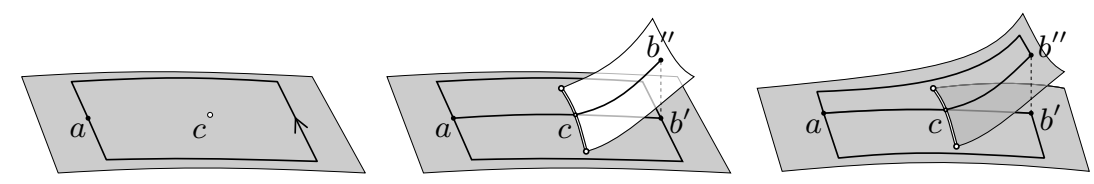

Figure 2. A simple $\mathcal{B D}$ of a system with monodromy (left), and cell unfolding surfaces with two overlapping lower cells of a system with nonlocal monodromy (centre), and with a single self-overlapping cell of a system with bidromy (right). Point $c$ in the leftmost image is an isolated critical value which lifts to a pinched torus; other points and paths are similar to those in figure 1.

1 at which the rank of $\partial(N, \mu, \mathcal{H}) / \partial(q, p)$ is non-maximal and critical fibres which contain such points. Regular fibres of our systems are 3 -tori $\mathbb{T}^{3}$; critical fibres can be smooth lower dimensional tori $\mathbb{T}^{2}$ or $\mathbb{S}^{1}$, a single point, or singular fibres of dimension 3 , such as singly or doubly pinched torus, curled torus, bitorus, etc. These singular fibres can be represented as direct products of the $\mathbb{S}^{1}$ cycle defined by the Keplerian symmetry action and certain two-dimensional singular fibers, which are depicted, for example, in (Cushman \& Bates, 1997, chapter IV.3, figure 3.5), (Nekhoroshev et al., 2006, Appendix A), and (Cushman \& Sadovskií, 1999; Efstathiou et al., 2007; Efstathiou, 2004). In particular, a pinched 2torus is obtained from a regular 2-torus by contracting one of its basic cycles to a point, which becomes a focus-focus equilibrium; a doubly pinched torus is a similar fibre with two pinch points. A bitorus is formed by two 2-tori glued together along a common basic cycle $^{2}$ which is a hyperbolic relative equilibrium, see fibre $c$ in figure 1.

In the cases we discuss below, the range of (2.1) is a simply connected domain $\bar{R}_{\mathcal{E M}}$ in $\mathbb{R}^{3}$. It is the closure of the set $R_{\mathcal{E M}}$ of all regular $\mathcal{E M}$ values, which can consist of several disjoint open subdomains. If within $\bar{R}_{\mathcal{E} \mathcal{M}}$ we distinguish strata of $\mathcal{E M}$ values with qualitatively different inverse images, and in particular if we distinguish critical and regular $\mathcal{E M}$ values, such $\bar{R}_{\mathcal{E M}}$ becomes a bifurcation diagram $\mathcal{B D}$ (Bolsinov \& Fomenko, 2004), which we can use to describe deformations (and in particular-bifurcations) of regular fibres under the variation of dynamical parameters $(n, m, h)$. For example, in figure 1 we follow the deformation of a regular fibre $\Lambda_{a}$ into two fibres $\Lambda_{b^{\prime}}$ and $\Lambda_{b^{\prime \prime}}$ along the path (acb); the singular fibre $\Lambda_{c}$ is a bitorus.

Description of the $\mathcal{B D}$ geometry involves the concepts of lower cell, unfolded lower cell, and cell unfolding surface, which are important in situations where preimages $\mathcal{E M}^{-1}(n, m, h)$ consist of several fibres (Sadovskií \& Zhilinskií, 2007). Lower and upper cells, and the cell structure of the phase space are introduced by Nekhoroshev et al. (2006). Upper cells are the closures of connected sets in the phase space (in our case $\mathbb{R}^{6}$ ) of regular fibers of the integrable map. They overlap only on their boundaries called walls. Lower cells are images of upper cells under the $\mathcal{E} \mathcal{M}$ map. They can overlap and self-overlap in $\bar{R}_{\mathcal{E M}}$, while in the unfolding surface $\bar{S}_{\mathcal{E M}}$, unfolded lower cells self-overlap and overlap each other only on their boundaries which consist of critical $\mathcal{E M}$ values. The open set of regular $\mathcal{E M}$ values in the interior of an unfolded lower cell is connected but not necessarily simply connected. The surface $\bar{S}_{\mathcal{E M}}$ can be constructed as a branch covering of $\bar{R}_{\mathcal{E M}}$, whose smooth sheets may be glued together along certain cell boundaries called branching walls. Several examples are shown in figures 1 and 2.

The study of individual unfolded bifurcation diagrams $\mathcal{B D}$ is naturally expanded to the description of parametric families of such $\mathcal{B D}$ 's. In this context, we prefer calling the latter

2 When the momentum $\mu$ defines a global $\mathbb{S}^{1}$ action which can be used to define a 'fixed' cycle $\gamma_{0}$ on all fibers, bitori can be further classified with regard to $\gamma_{0}$. We do not use such detailed classification in this paper. 
stratified $\mathcal{E} \mathcal{M}$ ranges or unfolding surfaces $\overline{\mathrm{S}}_{\mathcal{E} \mathcal{M}}$ in order to avoid confusing expressions, such as 'bifurcation of unfolded bifurcation diagram'. We describe a family of stratified $\mathcal{E M}$ ranges $\mathcal{B D}$ by specifying deformations and qualitative changes of $\mathcal{B D}$ under the variation of the external physical parameters $G, F_{b}$, and $F_{e}$.

5 Definition 1. Any two stratified $\mathcal{E M}$ ranges $\mathcal{B D}=\overline{\mathrm{S}}_{\mathcal{E M}}$ are called equivalent (or isomorphic) if they can be related (in an ambient space of the unfolding) by a smooth deformation.

Using this equivalence and the definition below, we can characterise the whole family of perturbed systems with Hamiltonian (1.1)

Definition 2. Perturbed Kepler systems with Hamiltonian (1.1) which can be approximated by integrable systems with integrable maps (2.1) and stratified $\mathcal{E M}$ ranges $\mathcal{B D}=\overline{\mathrm{S}}_{\mathcal{E} \mathcal{M}}$ are considered to be qualitatively equivalent if their $\mathcal{B D}$ are isomorphic. physical systems is quite comprehensive. So we provide only a very brief account here

3 in two degrees of freedom, by the geometric monodromy theorem of Cushman \& Duistermaat (2001); Vū Ngọc (2000); Zung (1997), a system has monodromy if it has an isolated critical $\mathcal{E M}$ value $c$ surrounded by regular $\mathcal{E M}$ values (figure 2, left), and the preimage $\mathcal{E M}^{-1}(c)$ of $c$ is a pinched torus. 

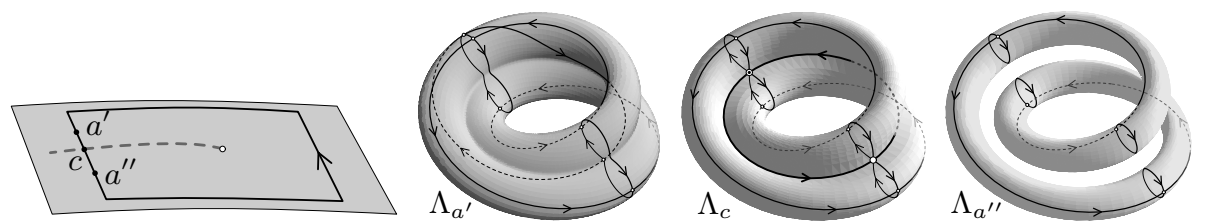

Figure $3 . \mathcal{B D}$ of a system with fractional monodromy (left, cf figure 2 left) with a line of weakly critical $\mathcal{E M}$ values $c$ (dashes) that lift to curled tori $\Lambda_{c}$. Fractional monodromy corresponds to the closed directed path (solid bold line) which goes around the strongly critical value (open circle) and crosses the critical line at $c$. Further plots illustrate the deformation of the regular fibres $\Lambda_{a^{\prime}}$ and $\Lambda_{a^{\prime \prime}}$ and of the cycles on them as we go from $a^{\prime}$ to $a^{\prime \prime}$ (from Nekhoroshev et al. (2006)).

\begin{abstract}
Monodromy is a mapping from the fundamental group $\pi_{1}$ of $R_{\mathcal{E M}}$ to the group of automorphisms of the first homology group $H_{1}\left(\mathbb{T}^{k}\right)$ of regular fibres which is isomorphic to the regular lattice $\mathbb{Z}^{k}$ (in our case $k=3$ ). It can be computed by choosing a closed directed path $\Gamma \subset \mathrm{R}_{\mathcal{E M}}$ (as, for example, in figure 2, left) and studying the connection on the torus bundle over $\Gamma$ induced by the local action-angle variables. The result depends only on the homotopy class of $\Gamma$ and is expressed using a matrix in $\operatorname{SL}(k, \mathbb{Z})$ which depends, naturally, on the basis choice in $H_{1}\left(\mathbb{T}_{a}^{k}\right)$ for some $a \in \Gamma$.

To follow the rest of this note, it is useful to recall that as a topological property, monodromy persists under continuous deformations of the system. This aspect and the related sign and addition theorems are exploited in the analysis in $\S 4 \mathrm{~b}$. In $\S 4 \mathrm{c}$ we give the first physical examples of generalised or fractional monodromy (Nekhoroshev et al., 2002, 2006; Efstathiou et al., 2007) as well as bidromy (Sadovskií \& Zhilinskií, 2007) which remained abstract concepts until now. Fractional monodromy generalises monodromy to a wider class of paths $\Gamma$ which intersect lines of particular 'weakly' critical values $c$. Over each $c$, the singular fibre $\Lambda_{c}$ (factored in our case by the Keplerian $\mathbb{S}^{1}$ action) has the topology of a twisted cylinder over figure eight and is called curled torus. The transformation of the regular tori in the neighbourhood of $\Lambda_{c}$ that occurs as we follow $\Gamma$ is shown in figure 3. Bidromy goes beyond the analysis of $\pi_{1}\left(\mathrm{R}_{\mathcal{E M}}\right)$ by associating automorphisms of $H_{1}\left(\mathbb{T}^{k}\right)$ with certain bipaths in the stratified $\mathcal{E M}$ range, such as the one in figure 2, right. Finally, since we deal with a quantum system, we imply constantly the correspondence (Cushman \& Duistermaat, 1988; Vū Ngọc, 1999; Sadovskií \& Zhilinskií, 1999) of classical Hamiltonian monodromy to defects (Zhilinskií, 2005; Nekhoroshev et al., 2006) of the lattice formed by the joint spectrum of quantum operators $(\hat{N}, \hat{\mu}, \hat{\mathcal{H}})$, a phenomenon also known as quantum monodromy. In fact, a computation of such spectrum by Schleif \& Delos (2007) was the principal source of motivation for this work.
\end{abstract}

\title{
3. Resonance zones in the parameter space
}

The parameter space $\mathcal{C}_{F G}$ of (1.1) is the set of relative configurations of 3-vectors $\mathbf{F}$ and $\mathbf{G}$ of respective lengths $F$ and $G$ that are not equivalent under rotations in $\mathrm{SO}(3)$. From $\langle\mathbf{F}, \mathbf{G}\rangle^{2} \leq G^{2} F^{2}$ we find that $\mathcal{C}_{F G}$ can be immersed in the positive quadrant of $\mathbb{R}^{3}$ with coordinates $F^{2}, G^{2}$, and $\langle\mathbf{F}, \mathbf{G}\rangle$ as a filled cone shown in figure 4. Strata of $\mathcal{C}_{F G}$ represent systems with different symmetries: the origin 0 corresponds to the unperturbed system, the open semiaxes $F^{2}>0$ and $G^{2}>0$ of the boundary $\partial \mathcal{C}_{F G} \backslash 0$ represent respective single-field Stark and Zeeman perturbations, other points of $\partial \mathcal{C}_{F G} \backslash 0$ represent parallel fields, while points in the open quarterplane $\left\{\langle\mathbf{F}, \mathbf{G}\rangle=0, F^{2}>0, G^{2}>0\right\}$ correspond to orthogonal fields, and the remaining interior points form a generic stratum. We further 

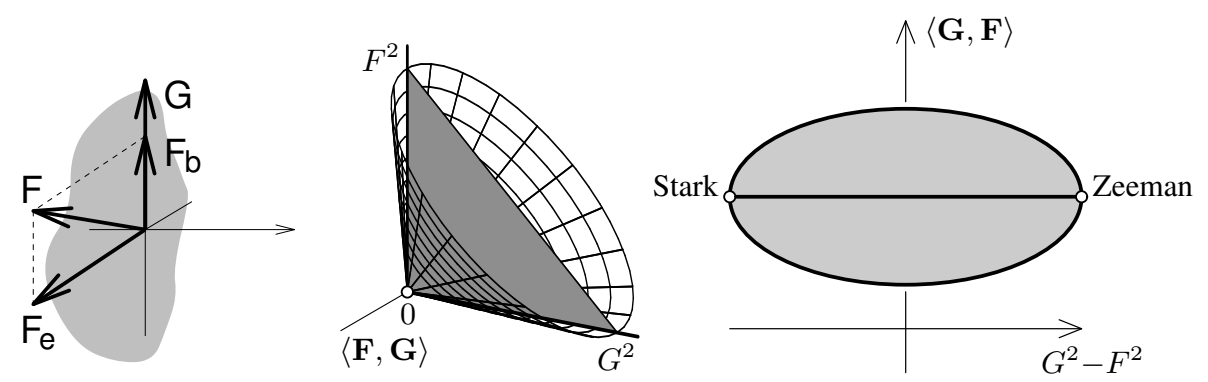

Figure 4. Electric and magnetic fields $\mathbf{F}$ and $\mathbf{G}$ (left), the set of all their distinct configurations (centre), and its constant $s$ section (right).

notice that the parallel stratum is a disjoint union of two open sets representing parallel and antiparallel configurations and that the generic stratum is also split in two halves with $\langle\mathbf{F}, \mathbf{G}\rangle>0$ and $\langle\mathbf{F}, \mathbf{G}\rangle<0$. It can be shown that systems which differ only in the sign of $\langle\mathbf{F}, \mathbf{G}\rangle$ have different energies but are otherwise qualitatively the same. So we can assume $\langle\mathbf{F}, \mathbf{G}\rangle \geq 0$.

The parameter space $\mathcal{C}_{F G}$ is further stratified into sets representing different $k_{+}: k_{-}$ resonant integrable approximations outlined in $\S 1$. Reducing the Keplerian symmetry, we obtain a reduced Hamiltonian $H_{n}=H_{0}+n H_{1}+n H_{2}+\ldots$ as function of six Keplerian invariants, the components of $\mathbf{L}$ and $\mathbf{K}$, which generate the Poisson algebra so(4) and which are bound by the relations $\langle\mathbf{K}, \mathbf{L}\rangle=0$ and $\mathbf{K}^{2}+\mathbf{L}^{2}=n^{2}$. Note that $N$ is the Casimir of the above algebra, and that the unperturbed Hamiltonian corresponds to $H_{0}=2 n$. The relations between $\mathbf{K}$ and $\mathbf{L}$ imply that the reduced phase space is $\mathbb{S}^{2} \times \mathbb{S}^{2}$. The Keplerian normal form $H_{n}$ contains an overall factor $n$, which, as can be shown, reflects the presence in (1.1) of a sole singular term $|\mathbf{Q}|^{-1}$. After rescaling by $n$, the lowest nontrivial order (i.e., the first average of the first order perturbation)

$$
H_{1}=g L_{1}-f_{b} K_{1}-f_{e} K_{2}
$$

in $H_{n} / n$ is linear in $(\mathbf{K}, \mathbf{L})$ and has, therefore, a linear Hamiltonian flow. Here

$$
g=G(2 / \Omega)^{2}, \quad f=3 F(2 / \Omega)^{3}, \quad\left(f_{e}, f_{b}\right)=3\left(F_{e}, F_{b}\right)(2 / \Omega)^{3},
$$


We now analyse the linear system with Hamiltonian (3.1) for fixed $s>0$ and all admissible values of $a$ and $d$. Rewritten in terms of the components of 3 -vectors

$$
\mathbf{X}=\frac{1}{2} \operatorname{diag}\left(R_{\alpha_{-}}, 1\right)(\mathbf{L}+\mathbf{K}) \quad \text { and } \quad \mathbf{Y}=\frac{1}{2} \operatorname{diag}\left(R_{\alpha_{+}}, 1\right)(\mathbf{L}-\mathbf{K}),
$$

where $\cos \alpha_{ \pm}=\left(g \pm f_{b}\right) / \omega_{ \pm}$and $\sin \alpha_{ \pm}= \pm f_{e} / \omega_{ \pm}$with

$$
\omega_{ \pm}=\sqrt{\left(g \pm f_{b}\right)^{2}+f_{e}^{2}}=s \sqrt{1 \pm 2 d},
$$

and $R_{\alpha}$ is the standard $2 \times 2$ matrix of counterclockwise rotation in a plane by angle $\alpha$,

$$
R_{\alpha}=\left(\begin{array}{rr}
\cos \alpha & -\sin \alpha \\
\sin \alpha & \cos \alpha
\end{array}\right)
$$

this Hamiltonian is ${ }^{5}$

$$
H_{1}=\omega_{-} X_{1}+\omega_{+} Y_{1} .
$$

Note that $\mathbf{X}^{2}=\mathbf{Y}^{2}=\frac{1}{4} n^{2}$ and that $\mathbb{S}^{2} \times \mathbb{S}^{2}$ can be regarded as the product of the ' $\mathbf{X}$-sphere' $\mathbb{S}_{X}^{2}$ and the ' $\mathbf{Y}$-sphere' $\mathbb{S}_{Y}^{2}$. Furthermore, components of $\mathbf{X}$ and $\mathbf{Y}$ define a standard Poisson algebra so(3) $\times \mathrm{so}(3)$ on this $\mathbb{S}^{2} \times \mathbb{S}^{2}$, so that the flow of (3.5) defines an $\mathbb{S}^{1}$ action which is a simultaneous rotation of $\mathbb{S}_{X}^{2}$ and $\mathbb{S}_{Y}^{2}$ about axes $X_{1}$ and $Y_{1}$ by angles $\omega_{-} t$ and $\omega_{+} t$ respectively.

Definition 3. The perturbed hydrogen atom system with Hamiltonian (1.1) is in $k_{-}: k_{+}$ resonance of order $k=k_{-}+k_{+}$when

$$
k_{-} \omega_{+}=k_{+} \omega_{-}, \quad \text { with } k_{ \pm} \in \mathbb{Z}_{>0} \quad \text { and } \operatorname{gcd}\left(k_{+}, k_{-}\right)=1 .
$$

So for a $k_{-}: k_{+}$resonant perturbation we have

$$
\frac{\omega_{-}}{k_{-}}=\frac{\omega_{+}}{k_{+}}=\omega=\sqrt{\frac{2\left(g^{2}+f^{2}\right)}{k_{-}^{2}+k_{+}^{2}}}=\frac{s \sqrt{2}}{\sqrt{k_{-}^{2}+k_{+}^{2}}}=\frac{s}{\kappa}
$$

${ }^{5}$ alternatively, $H_{1}$ can be represented in rotated Kustaanheimo-Stiefel coordinates (Cushman \& Sadovskií, 2000; Efstathiou et al., 2004) as a harmonic 4-oscillator Hamiltonian with frequencies $\pm_{-} \omega_{-}$and $\pm \omega_{+}$.

6 it can be shown that $\mathcal{H}$ is a polynomial in $n, X_{1}, Y_{1}$, and $\theta_{1}$, while $\theta_{2}$ enters only in the Euler-Poisson equations of motion; for the resonance of order $k, \theta_{1}$ and $\theta_{2}$ are of total degree $k$ in components of $\mathbf{X}$ and $\mathbf{Y}$. Notice that $\theta$ is chosen so that $\theta_{1}$ and $\theta_{2}$ for $k_{ \pm}=1$ agree with $\pi_{2}$ and $\pi_{3}$ of Cushman \& Sadovskií (2000). 

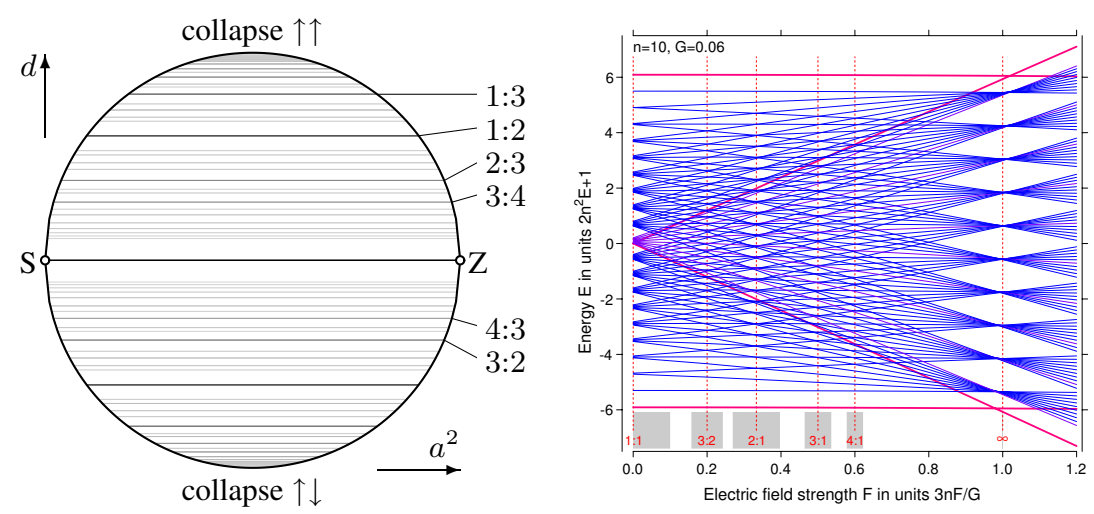

Figure 5. Systems with $k_{-}: k_{+}$resonances in the constant $s$ section (left) of the set of all possible perturbations of the hydrogen atom by static electric and magnetic fields $\mathbf{F}$ and $\mathbf{G}$. Manifestation of $k_{-}: k_{+}$resonances in the $n$-shell energy level structure of the parallel field system between the Zeeman limit and collapse (right, taken from (Sadovskií et al., 1996) with modifications). Fine and bold solid lines show energies of quantum levels and of four Keplerian RE respectively.

On the other hand, since characteristics, such as monodromy, used in definition 2 are topological in nature, they are continuous under sufficiently small deformations. As a consequence, we should be able to classify within the same family any exact $k_{-}: k_{+}$resonant system and systems with linear frequencies

$$
\omega_{ \pm}=s \sqrt{1 \pm 2\left(d_{k_{-}: k_{+}}+\delta\right)} \approx s\left(k_{ \pm} \kappa^{-1} \pm \kappa k_{ \pm}^{-1} \delta+O\left(\delta^{2}\right)\right)
$$

for $k_{ \pm} \neq 0,|d|<\frac{1}{2}$ and $|\delta| \ll 1$, i.e., outside the collapse regions, and

$$
\left(\omega_{\mp}, \omega_{ \pm}\right) \approx s \sqrt{2}\left(\delta, 1-\frac{1}{2} \delta^{2}+O\left(\delta^{4}\right)\right) \quad \text { for } d= \pm \frac{1}{2} \text { and } 0 \leq \delta \ll 1 .
$$

6 in the collapse regions where one of $\omega_{ \pm}$nearly vanishes and the respective $k_{ \pm}$is zero. Approximating $\omega_{ \pm}$by $\omega k_{ \pm}$with reasonably small $k_{-}+k_{+}$, we rewrite (3.5) as

$$
H_{1}=\omega \mu+\epsilon\left(\delta ; k_{-}, k_{+}\right) \nu, \quad \epsilon \ll \omega,
$$

9 where $\epsilon$ depends on the detuning $\delta$ and the choice of the resonance, and

$$
\mu=k_{-} X_{1}+k_{+} Y_{1} \quad \text { and } \quad \nu=k_{-} X_{1}-k_{+} Y_{1}
$$

19 Definition 5. The set of all detuned $k_{-}: k_{+}$systems is called $k_{-}: k_{+}$zone. 
Naturally, systems within each zone can be classified on the basis of definition 2. Several further important aspects should be pointed out right away.

Conjecture 1. For any $k_{-}: k_{+}$and sufficiently small total perturbation $s$ in an open interval $\Sigma$ of $\mathbb{R}_{>0}$, the $k_{-}: k_{+}$resonance zone contains an open domain of $\mathbb{R}^{3}$. Specifically, for any $k_{-}: k_{+}$and $s \in \Sigma$ we can find a small interval $\Delta_{s} \ni 0$, such that any system with frequencies (3.7) and $\delta \neq 0$ in $\Delta_{s}$ can be described as a detuned $k_{-}: k_{+}$system.

We can see from (3.7) and figure 5, left, that for fixed $s>0$, zones correspond to horizontal stripes centred on the $k_{-}: k_{+}$resonance lines so that $\left|d-d_{k_{-}: k_{+}}\right| \leq \delta_{\max }$. Their width can be defined as $2\left|\delta_{\max }\right|$ when $k_{+}+k_{-}>1$ or $\delta_{\max }$ for collapse zones. Clearly, if $\delta_{\max }$ is finite, zones cover inadvertently many resonances of order higher than that of the zone resonance. (For example, the 1:1 zone would include all resonances of sufficiently large order and $\left|k_{-}-k_{+}\right| \ll k_{-}+k_{+}$.)

Conjecture 2. At any given small $s>0$ and Keplerian action $n>0$, resonances of sufficiently high order are not important for the qualitative classification of systems with Hamiltonian (1.1) in the sense of definition 2.

In practice, our qualitative classification uses the normal form $\mathcal{H}$ truncated at some degree $k$ in components of $\mathbf{X}$ and $\mathbf{Y}$, and any resonances of orders higher than $k$ are neglected automatically since their specific resonance terms $\theta_{1,2}$ do not appear in $\mathcal{H}$.

Conjecture 3. With growing ns $>0$, an increasing number of higher order resonances becomes important, while the widths of the zones become smaller.

Note that the analysis of the orthogonal fields system (Cushman \& Sadovskií, 1999, 2000), one of the first fundamental physical systems where monodromy was uncovered, relied on the assumption, which was later proven as a theorem by Rink (2004); Broer et al. (2007), that monodromy could be generalised to KAM systems via an integrable approximation obtained by normalisation. This theorem is necessary to study monodromy in practically all real physical systems, and in our context -in all exactly resonant $k_{-}: k_{+}$ systems. Our conjectures here introduce yet another assumption and we believe that they can be proven using techniques similar to those of Rink (2004); Broer et al. (2007).

To conclude and to encourage further physical and mathematical studies of zones, we like to draw attention to their very clear quantum manifestation, which has been de facto produced by Sadovskií et al. (1996), but has not been analysed neither there nor-to our knowledge-later. In our figure 5, right, we reproduce the correlation diagram of Sadovskií et al. (1996), which represents $n$-shell energy levels of parallel fields systems with different ratios of $3 n F / G$. Since $n \approx 2 / \Omega$, this ratio is equivalent to our $f / g$ and in the fixed- $s$ subspace of $\mathcal{C}_{f g}$ (the disks in figures 4 and 5) the $3 n F / G$ span of figure 5, right, corresponds to the segment of the parallel stratum between the Zeeman limit $Z$ and the $\uparrow \uparrow$ collapse point $g=f_{b}=f$. As we depart from $Z$ (where $m$-multiplets exhibit a visible second order Zeeman splitting), we can see that quantum energies diverge linearly with $f / g$ and reassemble periodically and in different ways into multiplets of nearly degenerated levels. The $k_{-}: k_{+}$resonant values of $f / g$, which are given by (3.6) and are indicated in figure 5 , right, by vertical dashed lines for several low order resonances, coincide perfectly with these structures. Furthermore, multiplet degeneracies also confirm these resonances. In each case we also have an interval of $f / g$ values, i.e., a zone, within which the particular degeneracy of energy levels is well pronounced. The endpoints of these zones correspond approximately to the $g / f$ values at which outer energy levels of neighbouring multiplets meet. It can be seen that zone widths decrease with increasing $k_{-}+k_{+}$. 


\section{Classification of perturbations of the hydrogen atom}

We now possess a general framework to classify all possible perturbations of the hydrogen atom by small static external fields. Here we give a number of concrete examples. In each case, we normalise the first reduced Hamiltonian $H_{n}: \mathbb{S}^{2} \times \mathbb{S}^{2} \rightarrow \mathbb{R}$ for the second time and then analyse the resulting integrable system with reduced energy $\mathcal{H}$. (Recall that $H_{n}$ is a function of $(\mathbf{X}, \mathbf{Y})$ with principal order (3.5) composed of momenta $X_{1}$ and $Y_{1}$ which define $\mathbb{S}^{1}$ rotations of respective individual spheres in $\mathbb{S}^{2} \times \mathbb{S}^{2}$.) Stratified $\mathcal{E} \mathcal{M}$ images of our systems have a number of common features. First note the images of four $\mathbb{S}^{1}$ relative equilibria (RE) or nonlinear normal modes of the Keplerian symmetry, also known as Kepler ellipses, which correspond to equilibria of $H_{n}$ (Sadovskií \& Zhilinskií, 1998). Keplerian RE with maximal $|m|$ at given $n$ are stable; other Keplerian RE can become complex unstable and in that case their preimage includes their stable and unstable manifolds which form some kind of a pinched torus. Typical points on the external boundaries of the individual lower cells in the unfolding surfaces $\overline{\mathrm{S}}_{\mathcal{E M}}$ represent $\mathbb{T}^{2} \mathrm{RE}$ of the combined action of $\mathbb{S}^{1}$ symmetries associated with momenta $N$ and $\mu$; points on the branching walls represent bitori. Regular values lift to regular $\mathbb{T}^{3}$ or to two $\mathbb{T}^{3}$ for overlapping cells.

\section{(a) Nonresonant perturbations}

We consider first what happens when resonances are not important. This is generally possible for low $n s$ and away from the 1:1 and collapse zones which are always present. When $\omega_{+}$and $\omega_{-}$are incommensurate, we can normalise $H_{n}$ with respect to both $\mathbb{S}^{1}$ symmetries of (3.5). The resulting $\mathcal{H}$ Poisson commutes with both $X_{1}$ and $Y_{1}$ and is a polynomial in $\left(X_{1}, Y_{1}\right)$. Its domain of definition is the closure $\bar{D}_{n}$ of the open square $D_{n}:=\left\{\left(x_{1}, y_{1}\right):\left|x_{1}\right|<\frac{1}{2} n,\left|y_{1}\right|<\frac{1}{2} n\right\}$. The Hamiltonian functions $\left(X_{1}, Y_{1}\right)$ define a momentum map of $\mathbb{S}^{2} \times \mathbb{S}^{2}$ onto $\bar{D}_{n}$ and serve as global actions: any point in $D_{n}$ represents a regular torus $\mathbb{T}_{n, x_{1}, y_{1}}^{3}$ whose basis cycles are defined by $\left(N, X_{1}, Y_{1}\right)$. The functions $(\mu, \mathcal{H})$ define the specific energy-momentum map $\mathcal{E} \mathcal{M}_{k_{-}: k_{+}}$with values $(m, h)$ which gives an immersion $\psi_{k_{-}: k_{+}}: \bar{D}_{n} \rightarrow \mathbb{R}^{2}$. Recalling $\S 2$, we realise that $\bar{D}_{n}$ is an unfolded lower cell. In the simplest case illustrated in figure 6, left, $\psi$ is a diffeomorphism; in other situations, the surface $\mathcal{H}\left(X_{1}, Y_{1}\right)$ can typically fold so that its projection on the $(m, h)$ plane is not injective and we have open domains in the range of $\mathcal{E} \mathcal{M}_{k_{-}: k_{+}}$where each point lifts to several points in $D_{n}$. Part of the boundary of these domains consists of caustics, or curves whose points represent regular fibres with extremal energy. Caustics may signal that the resonance is pertinent:

Proposition 4.1. Caustics in the image of the $k_{-}: k_{+}$energy momentum map are structurally unstable.

In fact, for any even very small $\epsilon \neq 0$, adding a $k_{-}: k_{+}$resonance term $\epsilon \theta_{1}$ to $\mathcal{H}$ destroys a caustic typically so that the latter is replaced by a boundary representing periodic orbits $\mathbb{S}^{1}$ and a branching line near that boundary representing bitori. This happens because any two regular fibres with the same $\mathcal{E M}$ image have the same energy and as we approach a caustic, they become very close in the phase space, thus opening the door for any however small resonance to destroy them. Under such resonance, regular $\mathbb{T}^{2}$ preimages of caustic points disappear leaving a pair of periodic orbits, or nonlinear modes. The $\mathcal{E M}$ image of the stable mode remains at the boundary, while that of the unstable mode moves inside; the stable and unstable manifolds of the unstable mode form a bitorus. 

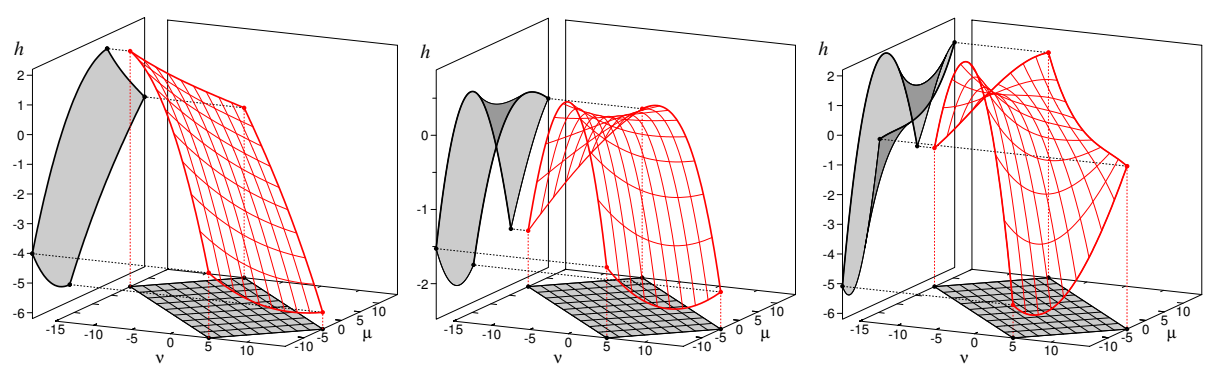

Figure 6. Example $\mathcal{B D}$ of nonresonant perturbations: simple regular $\mathcal{B D}$ (left), and self-overlapping $\mathcal{B D}$ with a caustic (centre,right).

\section{(b) Structure of the 1:1 zone}

The 1:1 resonance can never be ignored and its zone is quite large because the 1:1 resonance term $\theta_{1}$ appears in order $\mathcal{H}_{2}$ of the second normal form which comes immediately after the linear part $H_{1}$. In this note, we remain—for simplicity—at the level of $\mathcal{H}_{2}$.

Definition 6. Exactly resonant and detuned $k_{-}: k_{+}$systems that remain qualitatively unchanged in the sense of definition 2 under sufficiently small variations of field parameters $s>0, a$, and $d$ within the $k_{-}: k_{+}$zone are called structurally stable.

Definition 7. Equivalent (in the sense of definition 2) systems form a dynamical stratum within their zone.

In the parameter space $\mathcal{C}_{f g}$, dynamical strata can be represented similarly to the symmetry group action strata in figure 4 , centre. We describe all dynamical strata of structurally stable systems in the 1:1 zone which can be characterised using $\mathcal{H}_{2}$. To find these strata we study systems with different parameters $(a, d)$ within the zone using the standard techniques in (Cushman \& Bates, 1997; Cushman \& Sadovskií, 2000; Efstathiou et al., 2004), notably considering the topology of the families of energy levels of the reduced Hamiltonian. Notice that the classes of integrable Hamiltonian systems, which we discuss in this section, are quite typical. Thus all of them were described earlier on the example of the quadratic spherical pendulum (Efstathiou, 2004, chapter 4.2 and figure 4.2); Waalkens et al (2004) discussed similar systems.

The dynamical stratification of the $1: 1$ zone remains unchanged within a large interval of small $s>0$ because $\theta_{1}$ is part of $\mathcal{H}_{2}$. So we can work with constant- $s$ slices of $\mathcal{C}_{f g}$, such as the one in figure 4, right, where the 1:1 zone can be represented as a stripe centred on the $S Z$ line $\{d=0, a \in[0,1]\}$, see figure 7 , left. Within this stripe, various strata correspond to points, open segments, or open 2-domains. The latter represent structurally stable systems $A_{0}, A_{1}, B_{1}$, and $A_{1,1}$ (figures 7 , right, and 8) and are of primary interest to us. We describe also open segments $A_{2}$ and $B_{0}$ of $S Z$ which represent typical systems within the class of systems with an extra $Z_{2}$ symmetry. Notice that figure 7 , right, shows only half of the $1: 1$ zone with $d \geq 0$ because all strata are symmetric with respect to the $S Z$ axis. However, since each of the strata $B_{0}, B_{1}$, and $A_{1}$ has two disjoint parts, one near $S$ and another near $Z$, we distinguish such parts by prime and double prime respectively.

The $\mathcal{H}_{2}$ description of the dynamical stratification of the $1: 1$ zone can be summarised in the form of the genealogy graph in figure 7, right. Vertices of this graph represent (connected parts of) dynamical strata and edges correspond to typical paths along which structurally stable systems can be deformed from systems of one class into systems of another 

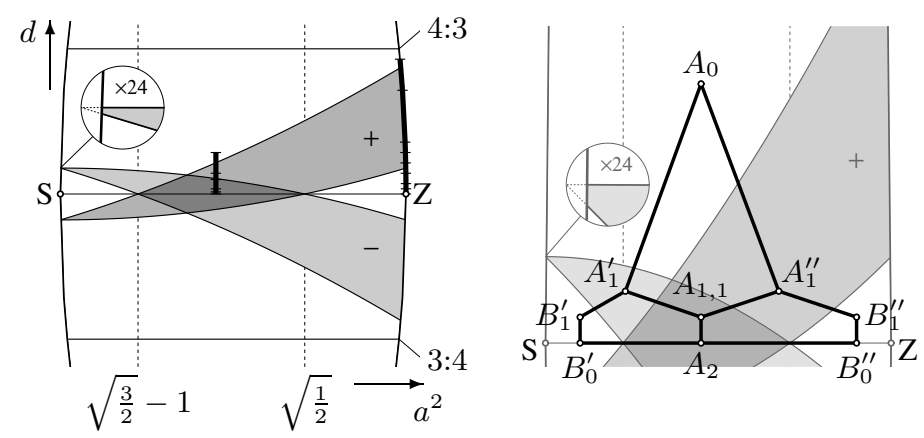

Figure 7. Structure of the 1:1 zone. Different dynamical strata of the zone (left) correspond to vertices of the genealogy graph (right). Vertical edges of the graph represent bifurcations with broken symmetry of order 2, other edges correspond to Hamiltonian Hopf bifurcations. Two bold paths (left) define $\mathcal{B D}$ families of detuned 1:1 systems; small ticks on the paths mark individual $\mathcal{B D}$ in figure 8 .

class. In a constant $s$ section of $\mathcal{C}_{f g}$, adjacent vertices correspond to open connected domains which share a common boundary $\sigma$. A typical path $\gamma$ which joins these domains, intersects $\sigma$ transversely in a single generic boundary point $c$ of $\sigma$ which corresponds to a bifurcation. Any small deformation of $\gamma$ does not change the family of systems it defines.

The $\mathcal{H}_{2}$ approximation is in some cases insufficient to remove the degeneracy of bifurcations represented by the edges of the $\mathcal{H}_{2}$ graph in figure 7, right. Specifically, $A_{0} A_{1}$, $A_{1} A_{1,1}$, and $A_{1} B_{1}$ represent Hamiltonian Hopf bifurcations (van der Meer, 1985; Duistermaat, 1998; Hanßmann \& Van der Meer, 2005) which can be fully characterised only after going to order $\mathcal{H}_{3}$, while the analysis of $A_{2} B_{0}$ given by Efstathiou et al. (2004) requires 10 $\mathcal{H}_{4}$. For some of these bifurcations, the small neighbourhood of the boundary between the ${ }_{1} \mathcal{H}_{2}$ strata may be further stratified. We do not resolve such possible fine structures here.

\section{(i) Exactly 1:1 resonant systems}

Exactly 1:1 resonant systems, i.e., perturbations by strictly orthogonal fields, have a special discrete symmetry $Z_{2}$ of order 2 , which is a composition of rotation by $\pi$ about axis $\mathbf{F}$ and reflection in the plane spanned by the vectors $\mathbf{F}$ and $\mathbf{G}$, see figure 4 and discussion by Sadovskií \& Zhilinskií (1998); Cushman \& Sadovskií (2000). These systems belong to a separate one-dimensional stratum $S Z$ of the symmetry group action in the middle of the 1:1 zone, the specific feature of the $1: 1$ zone. The $\mathcal{H}_{2}$ description of the dynamical stratification of $S Z$ was given by Cushman \& Sadovskií $(1999,2000)$; finer details were analysed by Efstathiou et al. (2004). There are two principal dynamical strata $A_{2}$ and $B_{0}$; $A_{2}$ systems are represented by points with $a^{2} \in(\sqrt{3 / 2}-1, \sqrt{1 / 2})$, while $B_{0}$ systems correspond to points on both sides of this central interval (figure 7). The $Z$ point is singled out by symmetry, but not dynamically (at least at the $\mathcal{H}_{2}$ level) because the Zeeman limit system with $\mathbf{F}=0$ is of type $B_{0}$. On the contrary, the $S$ point is isolated in both senses.

The $A_{2}$ systems have monodromy. It is caused by the presence of an isolated singular fibre called doubly pinched torus (Cushman \& Sadovskií, 1999, 2000) whose image is given by the isolated critical $\mathcal{E M}$ value in figure 8, bottom left (for $d=0$ ). Up to conjugation in $\operatorname{SL}(3, \mathbb{Z})$, the matrix of this monodromy ${ }^{7}$ is $\operatorname{diag}\left(1,\left(\begin{array}{cc}1 & 0 \\ 2 & 1\end{array}\right)\right)$. The stratified $\mathcal{E M}$ image of a $B_{0}$ system is shown in figure 8 , bottom right. Its unfolding surface $\mathrm{S}_{\mathcal{E} \mathcal{M}}$ has

\footnotetext{
7 for any path $\Gamma$ (cf. figure 9 ) the cycle associated with the Keplerian $\mathbb{S}^{1}$ symmetry transforms trivially, and
} 
three lower cells and is equivalent to the one shown in figure 1, top left. In both figures, the overlapping images of two lower cells are shaded dark. Regular values in the overlap region (such as $b$ in figure 1 ) lift to two regular tori $\left(b^{\prime}\right.$ and $\left.b^{\prime \prime}\right)$. Corresponding doublet quantum states in the spectrum of the quadratic Zeeman effect were discovered by Herrick (1982) and were related shortly after to classical dynamics by Solov'ev (1982, 1983). The latter work can be considered a predecessor of all studies based on $\mathcal{H}_{2}$.

The Stark limit system (point $S, a=0$ ) is exceptional: it has no resonance term $\theta_{1}$ and its $\mathcal{B D}$ has a caustic. Other exceptional $1: 1$ systems correspond to $Z_{2}$ equivariant Hamiltonian Hopf bifurcations, which mark the transition between $B_{0}^{\prime}$ and $A_{2}$ (on the $S$ side) and $B_{0}^{\prime \prime}$ and $A_{2}$ (on the $Z$ side). According to Efstathiou et al. (2004); Efstathiou (2004), these transitions involve additional bifurcations. So on both sides, $B_{0}$ and $A_{2}$ are separated by tiny one-dimensional dynamical strata of 'transitional' systems which lie near the respective critical values $\sqrt{3 / 2}-1$ and $\sqrt{1 / 2}$ of $a^{2}$. Efstathiou et al. (2004) show BD's of such systems in the bottom right of their figures 7 and 6 .

\section{(ii) Detuned 1:1 resonant systems}

To learn about all possible detuned 1:1 systems, we traverse the 1:1 zone along the two paths which start in $A_{2}$ and $B_{0}$ as shown in figure 7. Figure 8 shows the two resulting $\mathcal{B D}$ families. Notice that the second path is chosen to start at $Z$ and to stay on the parallel fields stratum ${ }^{8}$. This is justified because systems in the resulting family are dynamically equivalent (in the sense of definition 2) to neighbouring detuned systems in the interior of the 1:1 zone. Skewing $\mathbf{F}$ and $\mathbf{G}$, we break the additional $Z_{2}$ symmetry and move off the $(S Z)$ stratum. As an immediate consequence, the $A_{2}$ and $B_{0}$ systems bifurcate into $A_{1,1}$ and $B_{1}$ respectively. In figure 7 , left, $A_{1,1}$ is shaded dark, and $B_{1}$ consists of two wedge-like white regions $B_{1}^{\prime}$ and $B_{1}^{\prime \prime}$ near $S$ and $Z$ respectively. We describe briefly the bifurcations $A_{2} \rightarrow A_{1,1}$ and $B_{0} \rightarrow B_{1}$.

In the case of $A_{2}$, the isolated critical fibre separates into two singly pinched tori with different energies, while the corresponding isolated critical value $o$ separates into two such values $o^{\prime}$ and $o^{\prime \prime}$ as illustrated in figure 9. We can see that the fundamental group $\pi_{1}$ of the constant- $n$ section of the set $\mathrm{R}_{\mathcal{E M}}\left(A_{1,1}\right)$ of the regular $\mathcal{E M}$ values of the detuned $A_{1,1}$ system has two nontrivial generators $\Gamma^{\prime}$ and $\Gamma^{\prime \prime}$, which encircle $o^{\prime}$ and $o^{\prime \prime}$ respectively, while $\pi_{1}$ of $\mathrm{R}_{\mathcal{E M}}\left(A_{2}\right)$ has only one nontrivial generator $\Gamma$ which encircles $o$. Notice that $\Gamma^{\prime}+\Gamma^{\prime \prime}=\Gamma$ encircles $o^{\prime}$ and $o^{\prime \prime}$ together. Since monodromy persists under small deformations, the images of $\Gamma \subset \mathrm{R}_{\mathcal{E M}}\left(A_{1,1}\right)$ and $\Gamma \subset \mathrm{R}_{\mathcal{E M}}\left(A_{2}\right)$ under the respective monodromy mappings are the same. On the other hand, monodromy maps both $\Gamma^{\prime}$ and $\Gamma^{\prime \prime}$ to the $\operatorname{diag}\left(1,\left(\begin{array}{ll}1 & 0 \\ 1 & 1\end{array}\right)\right)$ class, thus illustrating the 'sign' of Hamiltonian monodromy (Cushman \& Vũ Ngoc, 2002).

In the case of $B_{0}$, the surface $\bar{S}_{\mathcal{E M}}\left(B_{0}\right)$ with three cells (figure 1, top left) changes into $\overline{\mathrm{S}}_{\mathcal{E M}}\left(B_{1}\right)$ with two cells (figure 2 , centre) after the branching line detaches from the boundary and becomes a string of critical values inside the regular interior of an unfolded lower cell. The latter cell has non-local monodromy $\operatorname{diag}\left(1,\left(\begin{array}{ll}1 & 0 \\ 1 & 1\end{array}\right)\right)$. Note that both endpoints of the branching line of $\overline{\mathrm{S}}_{\mathcal{E M}}\left(B_{1}\right)$ lift to singular (nonsmooth) tori.

the cycle basis in $H_{1}\left(\mathbb{T}_{n, m, h}^{3}\right)$ can always be chosen so that the full $3 \times 3$ monodromy matrix has block-diagonal form $\operatorname{diag}(1, M)$ and the sign of the offdiagonal element of $M$ is positive

8 one reason for this choice is that many atomic physicists are well familiar with the studies of perturbations by parallel fields with $G \gg F$ which followed the work by Solov'ev $(1982,1983)$ 
path $A_{2} A_{11} A_{1}^{\prime \prime} A_{0}$ with fixed $a^{2}=0.45$

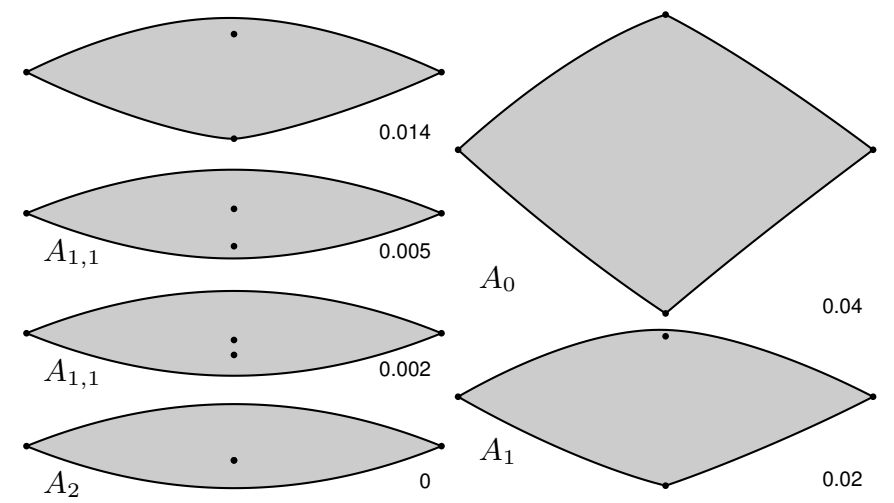

path $B_{0}^{\prime \prime} B_{1}^{\prime \prime} A_{1}^{\prime \prime} A_{0}$ for parallel fields near $Z$
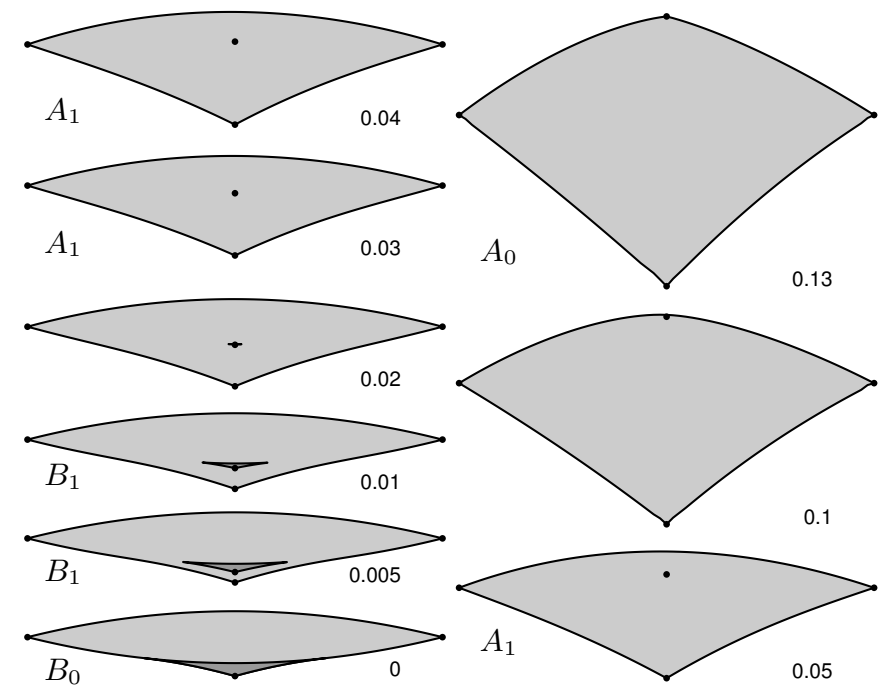

Figure 8. Changes (of the constant $n$ section) of the stratified $\mathcal{E M}$ images of the detuned 1:1 systems with $n s=0.1$ along the paths in figure 7, left. Value of the detuning parameter $d$ is displayed in the right bottom corner of each $\mathcal{E M}$ image plot. Filled circles mark Keplerian RE, solid and double lines show sets of $\mathbb{T}^{2} \mathrm{RE}$ and bitori, regular values are shaded gray, overlapping cells have a darker shade.

Transition to $A_{1,1}$ and $B_{1}$ occurs at arbitrarily small detuning $d \neq 0$. Further 'metamorphoses' of detuned 1:1 systems can be analysed quantitatively by computing the second normal form $\mathcal{H}$ and following the approach by Cushman \& Sadovskií (1999, 2000); Efstathiou et al. (2004). A fair idea of what goes on can be obtained by adding a small linear detuning term $d \nu$ to

$$
\mathcal{H}_{2}^{1: 1}=\frac{1}{8} s\left(1-2 a^{2}-2 a^{4}\right) \nu^{2}-\frac{1}{4} s a^{2} \theta_{1}
$$

computed by Cushman \& Sadovskií $(1999,2000)$ for the exact 1:1 resonance.

As we move along either of the paths in figure 7, left, and increase the detuning, our systems undergo several qualitative changes until they become a plain $A_{0}$ system. Each change involves a Hamiltonian Hopf bifurcation of one of the Keplerian RE with zero momentum $\mu$. The $\mathcal{B D}$ of the $A_{0}$ systems is a 'rectangle' whose four vertices represent $\mathrm{Ke}-$ 

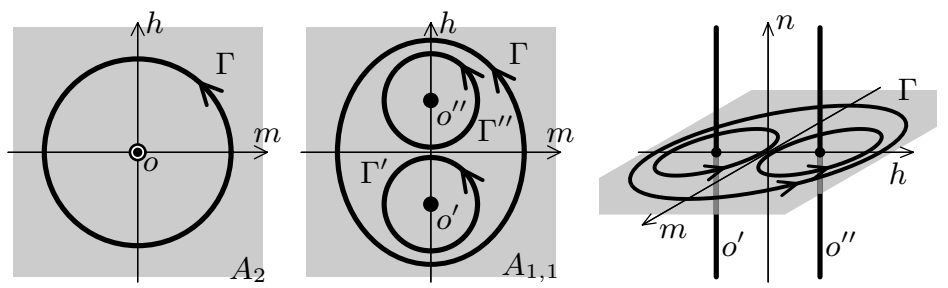

Figure 9. Contours in the stratified range of the energy-momentum map $\mathcal{E M}$ of the $A_{2}$ and $A_{1,1}$ systems in the 1:1 zone which encircle isolated critical values $o$ and $o^{\prime}$ and $o^{\prime \prime}$.
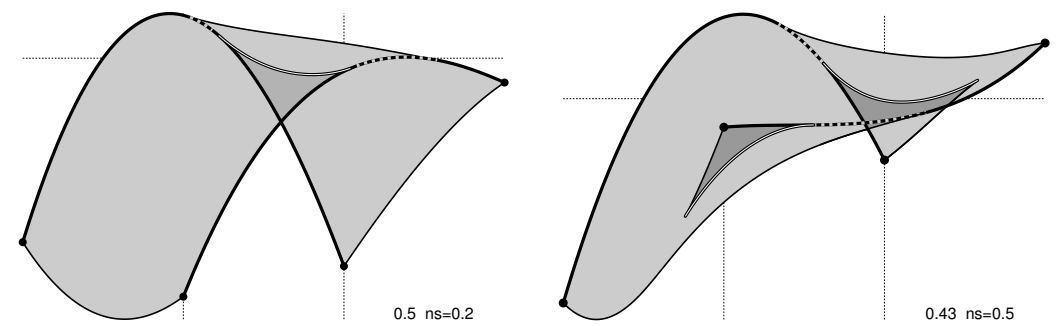

Figure 10. Stratified $\mathcal{E M}$ images of the 1:2 systems with fractional bidromy (left) and fractional monodromy (right). Bold dashes mark images of curled tori, other values are shown as in figure 8; energy scales are adjusted.

plerian RE and whose interior is regular and simply connected. Such systems have global actions and in some sense, reaching $A_{0}$ marks the outskirts of the zone where the resonance becomes unimportant (see $\S 4 \mathrm{a}$ and figure 6 , left).

We can see in figure 8 that before reaching $A_{0}$, systems $A_{1,1}$ and $B_{1}$ turn first into a system with one singly pinched torus represented by a single isolated critical value in the $\mathcal{E M}$ image. We call such systems $A_{1}$; their stratum consists of two parts $A_{1}^{\prime}$ and $A_{1}^{\prime \prime}$ shown by light gray shade in figure 7. We can further notice from this figure that $A_{1,1}$ can become either $A_{1}^{\prime}$ or $A_{1}^{\prime \prime}$ while $B_{1}^{\prime}$ and $B_{1}^{\prime \prime}$ turn into $A_{1}^{\prime}$ and $A_{1}^{\prime \prime}$ respectively. In the case of $A_{1,1}$ (see figure 8), one of its two unstable Keplerian RE becomes stable and the respective isolated critical value joins the boundary. The $B_{1}$ system turns into $A_{1}$ after a subcritical bifurcation, which occurs when the smaller triangular lower cell shrinks to a point and becomes an isolated critical value. At the last stage, the remaining isolated critical value of $A_{1}$ joins the boundary and $A_{1}$ becomes $A_{0}$.

\section{(c) Systems with higher resonances}

Unlike in the 1:1 systems, where the resonance term $\theta_{1}$ is part of the principal (quadratic) order $\mathcal{H}_{2}$ of the second normalised Hamiltonian, in systems with higher resonances, $\theta_{1}$ is relegated to order $\mathcal{H}_{k_{-}+k_{+}}$which is, typically, factor $(n s)^{k_{-}+k_{+}-2}$ smaller than $\mathcal{H}_{2}$. This means that the study of higher resonances is, essentially, a three-parameter problem, where different values of $n s$ should be considered along with those of $a$ and $d$. With growing $n s$, the contribution due to the $\theta_{1}$ term increases. This explains why higher resonances may become important only at sufficiently large $n s$. This also suggests that systems with higher resonances can be studied as blowups of caustics in the image of the $\mathcal{E M}$ maps (see $\S 4 \mathrm{a}$ ) which are obtained after truncating $\mathcal{H}$ at orders below $k_{-}+k_{+}$.

Another important difference from the 1:1 systems is the geometry of the reduced phase 
spaces $P_{m}$, or the spaces of orbits of the $k_{-}: k_{+}$resonant $\mathbb{S}^{1}$ action. When $k_{-}+k_{+}>2$, these spaces have cusp singularities which make the analysis of intersections $P_{m} \cap\{\mathcal{H}=$ $h\}$, the main tool in the construction of stratified $\mathcal{E M}$ images (Cushman \& Sadovskií, 2000; Efstathiou et al., 2004), highly nonlinear. As a consequence, any complete description of higher resonance zones, and in particular of the 1:2 zone, the largest and the most important of them, becomes significantly more involved and deserves a separate study.

In this note, we like to describe briefly two important typical representatives of exactly 1:2 resonant systems. These systems have two parameters, the field ratio $a$ with $a^{2} \in$ $\left[\frac{1}{10}, \frac{9}{10}\right]$ and the perturbation scale $n s$, which should be sufficiently smaller than 1 . Our computations show that the coefficient in front of $\theta_{1}$ in $\mathcal{H}_{3}$ is positive for all $a^{2}$ except for parallel fields when $a^{2}$ is $\frac{1}{10}$ or $\frac{9}{10}$ and the coefficient is zero. In comparison to the 1:1 case, the 1:2 systems are interesting due to the typical presence of specific 'weakly singular' fibres called curled tori (Nekhoroshev et al., 2002, 2006; Efstathiou et al., 2007). Their images under the $\mathcal{E M}$ map with fixed $n$ form typically strings $\sigma$ of critical values which Nekhoroshev et al. (2006) call 'passable' walls. Considering regular fibres $\mathbb{T}_{a}^{3}$ over a path $\Gamma \ni a \neq c$, which crosses such $\sigma$ (transversely) at $c$, we can continue certain full index-2 subgroups of first homology groups of $\mathbb{T}_{a}^{3}$ across the weak singularity $\mathcal{E M}^{-1}(c)$.

One type of 1:2 systems exists for relatively large and small values of $a^{2}$ in $\left(\frac{1}{10}, \frac{9}{10}\right)$, when the quadratic part $\mathcal{H}_{2}$ defines a well pronounced folded surface $\mathcal{H}(m, \nu)$ illustrated in figure 6 , centre. In the presence of $\theta_{1}$, the caustic in the energy-momentum projection of this surface blows up as shown in figure 10, left. We have a branching wall (double line) and a regular boundary (solid fine line) connected by two passable walls (dashed bold line). Neglecting, for the moment, the passable walls, this $\mathcal{B D}$ represents one self-overlapping unfolded lower cell of the type shown in figure 2, right. Hence we have a system with bidromy (Sadovskií \& Zhilinskií, 2007). The presence of passable walls signifies that we can only continue certain index-2 subgroups when we study this fractional bidromy.

When we fix $n s$ and sweep the interval of the remaining parameter $a^{2}$ starting at its maximum value (i.e., on the Zeeman side), we observe a distant similarity in the deformation of fixed-ns $\mathcal{B D}$ 's of exactly resonant 1:2 and 1:1 systems. In both cases, the energies of the two Keplerian RE with minimal absolute value $\frac{1}{2} n\left|k_{-}-k_{+}\right|$of momentum $\mu$ pass from the minimum to the maximum energy $h$ at given $n s$. For intermediate values of $a^{2}$, when the $\mathcal{B D}$ 'inverts' itself, we should expect complications.

In the 1:1 zone, these complications result in $A_{2}$ systems. In the 1:2 zone, different and somewhat more 'rare' systems are likely to exist for $a^{2}$ near 0.43 . According to our computations, the surface $\mathcal{H}_{2}\left(m, \nu ; a^{2}\right)$ nearly flattens at these values of $a^{2}$ and $\mathcal{H}_{3}$ becomes important even for moderate $n s$. The $\mathcal{B D}$ of such systems can be obtained after blowing up the caustic of the projected cubic surface in figure 6 , right, and is shown in figure 10 , right. Its unfolding surface has three sheets: a large main sheet to which two small triangular sheets called 'kites' or 'pockets', are glued along short branching lines. Each kite is a blowup of an ideal single point ending of the respective string of weakly critical values (bold dashes) 'attached' to it. Such ideal endings were studied by Nekhoroshev et al. (2002, 2006); Efstathiou et al. (2007), who introduced fractional monodromy with matrices in the class $\left(\begin{array}{rr}1 & 0 \\ -\frac{1}{2} & 1\end{array}\right)$ for a path $\Gamma$ which crosses the string once and encircles its endpoint. Kites are generic realisations of the same situation. By the usual deformation argument, monodromy for a path $\Gamma$, which lies in the main sheet, encircles one of the branching lines, and crosses the attached string of weakly critical values once, $\operatorname{should}$ be $\operatorname{diag}\left(1,\left(\begin{array}{cc}1 & 0 \\ \frac{1}{2} & 1\end{array}\right)\right)$. 


\section{Conclusion}

In the 80 years since Pauli's first attempt at classifying perturbations of the hydrogen atom by small and moderate static electric and magnetic fields (Pauli, 1926; van der Waerden, 1968; Valent, 2003), the progress in this area consisted of qualitative studies of particular members of this three-parameter family of systems, notably the discovery of vibrational and rotational dynamics in the Zeeman system (Herrick, 1982; Solov'ev, 1982), of the collapse (or 'crossover') limit (Sadovskií et al., 1996), and of monodromy in the orthogonal configuration (Cushman \& Sadovskií, 1999, 2000).

The implicit significance of the latter work was in showing essentially the way to the analysis of other perturbations. Unfortunately, this aspect remained underdeveloped by Cushman \& Sadovskií (1999, 2000); Efstathiou et al. (2004) and has not been appreciated duly. Without any appropriate framework and correct methodology, physicists were confined to very incomplete studies (Flöthmann et al., 1994; von Milczewski \& Uzer, 1997; Main et al., 1998; Berglund \& Uzer, 2001; Gekle et al., 2006). So one of our main goals here was to spell out the general approach to the classification of systems with Hamiltonian (1.1), based on the two-step normalisation, the equivalence relation in definition 2 , the appropriate choice of parameters, and the zone structure of the parameter space. Details on the techniques used in the analysis of resulting concrete integrable approximations within each zone can be found elsewhere (Cushman \& Bates, 1997; Efstathiou, 2004; Efstathiou et al., 2004; Michel \& Zhilinskií, 2001; Sadovskií et al., 1996; Cushman \& Sadovskií, 1999, 2000; Nekhoroshev et al., 2006; Efstathiou et al., 2007).

We ended the note by announcing a number of concrete results, notably a complete classification of 1:1 systems, and possible types of 1:2 systems, including the one with fractional monodromy. So pending a confirmation by quantum calculations and numerical simulations, hydrogen atom in fields will-like with the usual 'integer' monodromy in the earlier study by Cushman \& Sadovskií (1999, 2000)_-become the first known fundamental physical system with fractional monodromy. A full account of these studies will be published in a series of forthcoming papers.

We thank Professor John B. Delos and his post-graduate student Chris Schleif for drawing our attention to this system, and for sharing their preliminary results (Schleif \& Delos, 2007).

\section{References}

Berglund, N. \& Uzer, T. 2001 The averaged dynamics of the hydrogen atom in crossed electric and magnetic fields as a perturbed Kepler problem, Found. Phys. 31 283-326.

Bolsinov, A. V. \& Fomenko, A. T. 2004 Integrable Hamiltonian systems. Geometry, topology, classification. Boca Raton, Florida: Chapman \& Hall/CRC.

Broer, H. W., Cushman, R. H., Fassò, F. \& Takens, F. 2007 Geometry of KAM-tori for nearly integrable Hamiltonian systems, Ergod. Th. \& Dynam. Sys., to appear.

Cushman, R. H. \& Bates, L. 1997 Global aspects of classical integrable systems. Basel: Birkhäuser.

Cushman, R. H. \& Duistermaat, J. J. 1988 The quantum mechanical spherical pendulum, Bull. Am. Math. Soc. 19, 475-479. 
Cushman, R. H. \& Duistermaat, J. J. 2001 Non-Hamiltonian monodromy, J. Diff. Eq. 172, $42-58$.

Cushman, R. H. \& Sadovskií, D. A. 1999 Monodromy in perturbed Kepler systems: hydrogen atom in crossed fields, Europhys. Lett. 47, 1-7.

Cushman, R. H. \& Sadovskií, D. A. 2000 Monodromy in the hydrogen atom in crossed fields. Physica 142 D, 166-196.

Cushman, R. H. \& Vũ Ngọc, S. 2002 The sign of the monodromy for Liouville integrable systems, Ann. Inst. H. Poincaré 3, 883-894.

Duistermaat, J. J. 1980 On global action angle coordinates, Comm. Pure Appl. Math. 33, 687-706.

Duistermaat, J. J. 1998 The monodromy in the Hamiltonian Hopf bifurcation, Z. Angew. Math. Phys. 49, 156-161.

Efstathiou, K. 2004 Metamorphoses of Hamiltonian Systems with Symmetry. Lecture Notes in Mathematics, vol. 1864. Heidelberg: Springer-Verlag.

5 Efstathiou, K., Cushman, R. H. \& Sadovskií, D. A. 2004 Hamiltonian Hopf bifurcation of the hydrogen atom in crossed fields, Physica 194 D, 250-274.

7 Efstathiou, K., Cushman, R. H. \& Sadovskií, D. A. 2007 Fractional monodromy in the 1: -2 resonance. Adv. Math. 209, 241-273.

Flöthmann, E., Main, J. \& Welge, K. W. 1994 The Kepler ellipses of the hydrogen atom in crossed electric and magnetic fields, J. Phys. B: At. Mol. Opt. Phys. 27, 2821-2833.

Gekle, S., Main, J., Bartsch, T. \& Uzer, T. 2006 Extracting multidimensional phase space topology from periodic orbits, Phys. Rev. Lett. 97, 104101.

Hanßmann, H. \& Van der Meer, J. C. 2005 Algebraic methods for determining Hamiltonian Hopf bifurcations in three-degree-of-freedom systems, J. Dyn. Diff. Eqs. 17, 455-474.

Herrick, D. R. 1982 Symmetry of the quadratic Zeeman effect for hydrogen, Phys. Rev. A 26, 323-329.

Main, J., Schwacke, M. \& Wunner, G. 1998 Hydrogen atom in combined electric and magnetic fields with arbitrary mutual orientations, Phys. Rev. A 57, 1149-1157.

Michel, L. \& Zhilinskií, B. I. 2001 Rydberg states of atoms and molecules. Basic grouptheoretical and topological analysis, Phys. Rep. 341, 173-264.

von Milczewski, J. \& Uzer, T. 1997 Canonical perturbation treatment of a Rydberg electron in combined electric and magnetic fields, Phys. Rev. A 56, 220-231.

Nekhoroshev, N. N., Sadovskií, D. A. \& Zhilinskií, B. I. 2002 Fractional monodromy of resonant classical and quantum oscillators, C. R. Acad. Sci. Paris, Ser. I, 335, 985-988.

Nekhoroshev, N. N., Sadovskií, D. A. \& Zhilinskií, B. I. 2006 Fractional Hamiltonian monodromy, Ann. Henri Poincaré 7, 1099-1211. 
Pauli, W. 1926 Über das Wasserstoffspektrum vom Standpunkt der neuen Quantenmechanik. Z. Physik A 36, 336-363.

Rink, B. 2004 A Cantor set of tori with monodromy near a focus-focus singularity, Nonlinearity 17, 347-356.

Sadovskií, D. A., Zhilinskií, B. I. \& Michel, L. 1996 Collapse of the Zeeman structure of the hydrogen atom in the external electric field, Phys. Rev. A 53, 4064-7.

Sadovskií, D. A. \& Zhilinskií, B. I. 1998 Tuning the hydrogen atom in crossed fields between the Zeeman and Stark limits, Phys. Rev. A 57, 2867-84.

Sadovskií, D. A. \& Zhilinskií, B. I. 1999 Monodromy, diabolic points, and angular momentum coupling, Phys. Lett. 256A, 235-244.

Sadovskií, D. A. \& Zhilinskií, B. I. 2007 Hamiltonian systems with detuned 1:1:2 resonance. Manifestation of bidromy, Ann. Phys. (N.Y.) 232, 164-200.

Schleif, C. \& Delos, J. B. 2007 New features in the energy spectrum of hydrogen in crossed fields, submitted to Phys. Rev. A.

Solov'ev, E. A. 1982 The hydrogen atom in a weak magnetic field, Zh. Eksp. Teor. Fiz. 82, 1762-1771 (Transl. Sov. Phys. JETP 55, 1017).

Solov'ev, E. A. 1983 Second-order perturbation theory for the hydrogen atom in crossed electric and magnetic fields, Zh. Eksp. Teor. Fiz. 85, 109 (Transl. Sov. Phys. JETP 58, 63-66).

Valent, G. 2003 The hydrogen atom in electric and magnetic fields: Pauli's 1926 article, Am. J. Phys. 71, 171-175.

Van der Meer, J. C. 1985 The Hamiltonian Hopf bifurcation. Lecture Notes in Mathematics, vol. 1160. New York: Springer-Verlag.

Van der Waerden, B. L. 1968 Sources of Quantum Mechanics. New-York: Dover.

Vū Ngọc, S. 1999 Quantum monodromy in integrable systems, Comm. Math. Phys. 203, 465-479.

Vū Ngọc, S. 2000 Bohr-Sommerfeld conditions for integrable systems with critical manifolds of focus-focus type, Comm. Pure Appl. Math. 53, 143-217.

Waalkens, H., Dullin, H. R. \& Richter, P. H. 2004 The problem of two fixed centres: bifurcations, actions, monodromy, Physica 196 D, 265-310.

Zhilinskií, B. I. 2005 Interpretation of quantum Hamiltonian monodromy in terms of lattice defects, Acta Appl. Math. 87, 281-307.

Zung, N. T. 1997 A note on focus-focus singularities, Diff. Geom. Appl. 7, 123-130. 